\title{
2. X-RAY ASTRONOMY
}

K A Pounds

\section{A. INTRODUCTION}

The perlod leading up to the last IAU General Assembly was dominated in Xray Astronomy by the results from the Einstein Observatory. This first application of a large, satellite-borne, high resolution X-ray telescope to the study of cosmic sources had led, by the end of orbital operation in April 1981, to the detection of $\mathrm{X}$-ray fluxes from a wide variety of astronomical objects and the full maturing of $X$-ray Astronomy. During the past three years a strong scientific output has continued to flow from the analysis of the more than 5600 separate Einstein observations, many of which are now widely available via the Einstein Data Bank. In sharp contrast, the peak of activity and scientific output from Einstein will apparently be followed, in the United States, by almost a decade with no new X-ray satellite. Fortunately, successful launches have been achieved in both the Japanese and European programmes, with Tenma launched in February 1983 (to join Hakucho in orbit) followed by EXOSAT three months later. Both these new missions have attracted world-wide interest and observing time on both is at a premium. Of a range of instruments on board the Tenma satellite the most productive is proving to be the large $\left(\sim 800 \mathrm{~cm}^{2}\right)$ array of gas scintillation proportional counters. These detectors, flown for the first time on Tenma (and EXOSAT), provide a factor-of-two increase in spectral resolution over the more conventional proportional counters, an advantage which is particularly valuable in resolving the key emission line and absorption features associated with the K-shell of iron, near $\sim 7 \mathrm{keV}$. One particularly exciting result reported from this Tenma instrument was an apparent absorption feature at $\sim 4.1 \mathrm{keV}$ in X-ray burst spectra from the source 2S 1636-536 (later supported by EXOSAT observations), the feature being attributed to red-shifted iron absorption in the strong gravitational field close to the neutron star burst source! At the time of writing (December 1984) a power storage problem is limiting the operational efficiency of Tenma, but the scientific payload continues to function well.

The unique orbit of EXOSAT is proving to be a major asset, with continuous observations of more than 70 hours being possible, uninterrupted by Earth eclipse. In addition, the apogee over N. Europe allows real-time operation of EXOSAT, analogous to observations with a ground-based telescope. The scientific payload consists of an array of proportional counters (effective area $\sim 1600$ $\left.\mathrm{cm}^{2}\right)$, a small gas scintillation proportional counter $\left(\sim 90 \mathrm{~cm}^{2}\right)$ and 2 imaging telescopes. Good data have been obtained from all the EXOSAT instruments, but problems with some of the focal plane detectors and the mechanical drive are currently preventing the use of the second telescope and of either transmission grating. Given the considerable scientific potential and current scarcity of $X-$ ray spectra of corresponding resolution the latter is particularly disappointing. Nevertheless, to date over 1500 EXOSAT observations have been carried out, the programme continues to be heavily over-subscribed and it is hoped to continue full operation until satellite re-entry in 1987 .

An expanding effort in X-ray astronomy is also reported from the Soviet Union with the Salyut-7 orbital station carrying several experiments for $X-r a y$ astronomy. Of particular interest is the RT-4M instrument, a grazing incidence telescope with a non-imaging flow gas proportional counter as focal plane detector. The telescope optics are of two-fold nested wolter type I design, with a maximum entrance aperture $24 \mathrm{~cm}$ wide and a focal length of $66 \mathrm{~cm}$. The reflecting surfaces are coated with gold (outer mirror) and nickel (inner 
mirror) to provide an operating wavelength band between $44 \mathrm{~A}$ and $100 \mathrm{~A}$ over a field of view of $4^{\circ}$ by $3^{\circ}$. The detector entrance aperture is divided into three parallel sectors, viewing different fields (Mandelstam et al, 1983). Results from this and other Salyut instruments are eagerly awaited. In the following section, a (necessarily personal and incomplete) review is made of progress in X-ray astronomy over the period since the Commission's 1982 report.

\section{B. SCIENTIFIC ACHIEVEMENT}

\section{(i) Stellar emission.}

Einstein observations have provided an outline of the X-ray emission properties of stars throughout the H-R diagram (e.g. Vaiana et al, 1981; Swank et al, 1981; Caillault and Helfand, 1982; Montmerle et al, 1983), but a detailed understanding has so far been limited by the small sample of higher resolution spectra obtained with the Einstein grating or crystal spectrometers (e.g. Mewe et al, 1981; Swank and Cassinel11, 1983; Swank and Johnson, 1982; Vedder and Canizares, 1983). Evidence that stellar surface activity (as an effect of surface convection) does indeed cease along the main sequence is provided by the discovery that the X-ray luminosity decreases from a mean of $10^{29} \mathrm{erg} \mathrm{s}^{-1}$ at $\mathrm{F5}$ to upper limits of $\sim 10^{27} \mathrm{erg} \mathrm{s}^{-1}$ at $\mathrm{AO}$ (Schmitt et al, 1985). Early type stars are readily detectable as $X$-ray sources but it is not yet clear whether the emission is from coronae, shocked winds, or some other cause. Recent models have been discussed by Waldron 1981), Lucy and White (1980) and Lucy (1982), among others. Of particular value in determining the evolution of stellar X-ray emission is the study of star clusters (of common age), such as those in the Hyades (Stern et al, 1981) and Pleiades (Caillault and Helfand, 1985) Clusters. Preliminary indications are that for solar type stars the fall in $X-r a y$ output (at least up to $210^{9} \mathrm{yr}$ ) is slower than $t^{-0.5}$.

The significance of RS CVn stars as highly variable $\mathrm{X}$-ray sources was underlined in an analysis of short-lived Ariel-5 transients (Pye and McHardy, 1983), who found 6 of 11 optically identifled X-ray transients to be RS CVn systems. The long EXOSAT observing time is well matched to the study of such systems. For example, Barstow (1985) has observed the RS CVn binary HR1099, during which an X-ray outburst was followed through a peak (of $3 \times 10^{34.5} \mathrm{erg}^{-1}$, $2-6 \mathrm{keV})$ and subsequent decay over $\sim 2$ hours. Interesting, non-periodic modulations were also clearly seen. Other recent EXOSAT stellar observations have covered the contact binary VW Cephei, revealing a structured corona (Vilhu and Heise, 1985), the 12 day rotating K2 V star $\in$ Eri (Horstman et al, 1985), and the nearby triple star system Algol. Observations of Algol during the secondary optical eclipse ( $B$ star in front of $K$ Companion) have provided lower limits to the size of the $\mathrm{K}$ star's X-ray corona, while ME and grating spectra revealed a thermal spectrum with Fe XVII and XVIII emission 1 ines and a continuum temperature of $2.4 \times 10^{7} \mathrm{~K}$ (Culhane et al, 1985). A large X-ray flare was also seen, with an effective temperature of $6 \times 10^{7} \mathrm{~K}$ and iron emission up to Fe XXV (Parmar et al., 1985). Further detections of soft X-rays from hot white dwarf stars have been reported from Einstein observations (Kahn et al, 1984). EG 187, Gr 288, Gr 289 and LB 1663 have spectra consistent with DA white dwarf photospheric temperatures in the range $3-6 \times 10^{4} \mathrm{~K}$. Complementary IUE data show the need for some atmospheric opacity, with a $\mathrm{H}: \mathrm{He}$ ratio in the range (2-600) $\times 10^{-5}$. The implication of these detections is that many more white dwarf stars should be available for study in future XUV surveys.

\section{(ii) Supernova remnants.}

The Einstein Observatory has successfully mapped more than 40 galactic supernova remnants and has detected 25 and 6 , respectively, in the Large and Small Magellanic Clouds (Mathewson et al, 1983 and references therein). The 
best data naturally arise for the relatively young galactic remnants and detailed studies have been reported on a number, including Tycho's SNR (Seward et al, 1983), SN 1006 (Pye et al, 1981), Cas A (Stewart et al, 1983) and most recently RCW 86 (=SN185A.D.), observed with Einstein by Pisarski et al (1984). Other high quality X-ray maps have been obtained with Einstein for several. older, but bright, remnants, in particular Puppis A (Petre et al., 1982) and Cygnus Loop (Ku et al., 1984). These observations have generally supported the view that the $\mathrm{X}$-ray emission from these remnants is thermal, from shock heated gas (of the supernova itself and the surrounding interstellar medium) at temperatures $\sim 1-10 \mathrm{keV}$. Spatial structures have been compared with radio and optical features. X-ray spectra taken with the Einstein solid state spectrometer (see review by Holt, 1983) have been analysed in terms of thermal models, but, in general, the lack of spatial resolution and uncertainties as to the state of equilibrium of the emitting plasma have limited the reliability of the deduced mass and element abundance distributions. The high resolution crystal spectrometer data from Puppis A (Winkler et al, 1981) have shown the great promise of future, more sensitive observations. EXOSAT X-ray maps of Puppis A (Aschenbach et al, 1985), SN 1006 at higher resolution (Jones et al, 1984) and Cygnus Loop (Arnaud et al, 1985) are now adding to this field.

An increasing number of core-halo remnants have been reported, some with an associated neutron star. Four pulsars have been found in SNR to date, while Einstein observations have added a further six with point sources too weak to allow pulsations to be detected (Seward, 1983). In addition to the Crab Nebula, MSH15-52 has been found to contain an X-ray/radio pulsar with the highest known rotational energy loss rate (Seward and Harnden, 1982) and a further Einstein observation showed CTB109 to contain a long period ( $7 \mathrm{sec}$ ) X-ray pulsar (Fahlman and Gregory, 1983). Other remnants found from Einstein observations to have weak point X-ray sources include RCW103, 3C58, CTB80 and W28 (Tuohy and Garmire, 1980; Becker et al, 1982; Andrews et al, 1983). A recent EXOSAT addition to this sub-group is the southern SNR PKS1209-52 (Kellett, 1985).

\section{(iii) Galactic X-ray binaries with compact components.}

By their nature, both Tenma and EXOSAT are well suited to detailed study of the bright $X$-ray binaries containing white dwarf, neutron star or (possibly) black hole components. Over 100 of these sources now have identified optical counterparts (Bradt and McClintock, 1983) and it is conventional to divide them according to system mass, nature of the compact component, or location (e.g. globular cluster). An excellent review of this broad field is now publishd in Lewin and van den Heuvel (1983) while other reviews may be found in a special volume of Physica Scripta (vol. T7, 1984).

Recent progress in the study of massive $\mathrm{X}$-ray binaries (sometimes called class I) where the components are an early type star of $M>10 M_{0}$ and a neutron star has concentrated on the details of mass loss, stellar wind opacity, pulse structure etc., since this class is basically better understood than the low mass systems (most of which are not yet actually established to be binary). Continuing Hakucho and Tenma observations of Vela $X-1$ have now shown the spindown, noted in the Commissions's report 3 years ago, to have again reversed, indicating a complex interaction between the accreting material and the neutron star (Nagase et al, 1984 and references therein). The rate of change of the pulsar period in LMC X-4 is also in strong discord with a simple accretion torque model (Keller et al., 1983). Two of the best candidate X-ray binaries to contain a black hole, Cygnus $X-1$ and GX 339-4 remain 'unproven', though new high quality data has been obtained on the short term X-ray variability (e.g. Maejima et al, 1984; for GX339-4 and Meekins et al, 1984 and Page, 1985; for Cyg X-1). In addition, the analysis of SAS-3 data has revealed an intriguing series of intensity 'dips' generally occurring near superior conjunction of the $X$-ray 
source (Remillard and Canizares, 1984). Identification of several 'classical' $X$-ray transient sources with Be stars (Warwick et al, 1981; Rappaport and van den Heuvel, 1982; Steiner et al, 1984) has been followed by further detailed optical and X-ray studies and it now seems likely that it is the strong equatorial mass loss of the Be star that fuels the neutron star accretion over the large (and probably eccentric) orbits of such long period binaries as 114561 (187d; Watson et al., 1981), 4U0115+63 (24d) and the highly luminous LMC transient A0538-66 (16.6d; Charles et al, 1983).

The major recent advance in the understanding of low mass (or class II) systems has been the establishment of a binary period for several cases, typically of a few hours (though there are notable exceptions, e.g. Cygnus X-2 at 9.8 days). Thus $2 \mathrm{~A} 1822-371$ has been found to have a binary period of $5.6 \mathrm{~h}$ (Cowley et al, 1982 and references therein) and $2 \mathrm{~A} 2129+470$ one of $5.2 \mathrm{~h}$ (McClintock et al, 1982). The proposition that X-ray bursters are a sub-class of low mass binaries (having a lower mean accretion rate or weaker neutron star magnetic field?) has been justified by the identification of binary periods for 1636-53 ( $\sim 4 \mathrm{~h}$, from optical photometry; Pedersen et al., 1981), 1915-05 ( 50m, from X-ray dips; White and Swank, 1982), 1755-33 (4.4h, from X-ray dips; White et al., 1984), 1659-29 $(7.1 \mathrm{~h}$, from X-ray dips; Cominsky and Wood, 1984) and $1254-69(\sim 3.9 \mathrm{~h})$. The latter result is perhaps the most interesting in that the period is found from both optical photometry (Motch et al., 1984) and X-ray dips (Courvoisier et al., 1985). X-ray burst sources have continued to receive attention in the Tenma and EXOSAT programmes. Use of large detector arrays, with improved spectral and timing resolution, are yielding high quality data which are helping to clarify details of the mechanism and geometry of the emission region. The previously established model for the more common type I bursts, involving thermonuclear fusion on the surface of an accreting neutron star, remains intact, but only now are first attempts being made to calculate the radiation transfer properties in the neutron star photosphere. The intriguing detection of an apparently red-shifted absorption line by Tenma (Waki et al, 1984) and EXOSAT observers (Turner et al, 1984) has been mentioned previously. Correlated optical and X-ray bursts have been detected for 1735-44 (Grindlay et al., 1978), 1636-536 (Matsuoka et al., 1984) and - most recently for MXB1659-29 (Matsuoka et al, 1984).

Probably the most significant progress in the study of X-ray binaries during the past 3 or 4 years has been of those systems involving accretion onto a white dwarf star, the cataclysmic variables. Because of their proximity ( $~ 50-$ $500 \mathrm{pc}$ ) cataclysmic variables are excellent objects in which to study the physics of accretion onto a compact object. They are also readily studied by $x-$ ray techniques and some 50 have now been so detected (see review by Cordova and Mason, 1983). Ariel-5, HEAO-1 and Einstein observations have been actively followed up in the EXOSAT programme. An important variable in such systems, which are believed to involve a low mass star and a white dwarf, is the magnetic field strength of the dwarf star. Twenty or so AM Her (or polar) and DQ Her (intermediate polar) type stars have strong fields which, in the former case, lock the white dwarf rotation to the binary period. Most cataclysmics, such as SS Cyg and U Gem, however, show no evidence of magnetic fields. One of the earliest EXOSAT observations showed GK Persei to have a strongly modulated X-ray flux, establishing $351 \mathrm{sec}$ as the white dwarf rotation period and GK Per as the sixth known 'intermediate polar'. Extended observations further showed the depth of modulation to be independent of mean flux (accretion rate), indicating an extended, thin polar cap emission region (Watson et al, 1985a). Repeated EXOSAT observations of SS Cygni between and during optical outbursts have confirmed the anti-correlation of hard and soft X-ray flux (Watson et al, 1985) and are providing detailed information on the emission in such a non-magnetic white dwarf system, which appears to involve thermal radiation (of the soft component) from the surface of the white dwarf and a (harder) component from an 


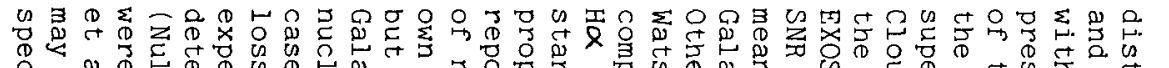

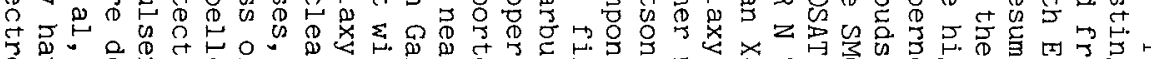

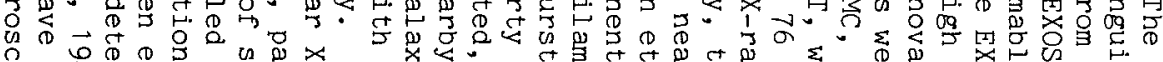

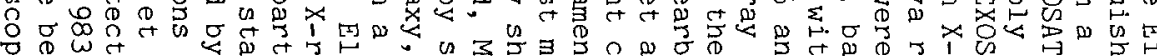

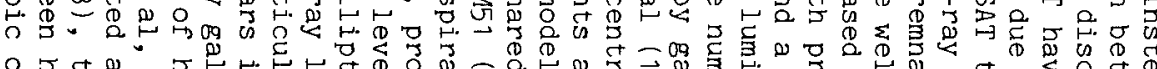

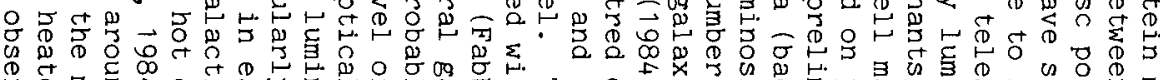

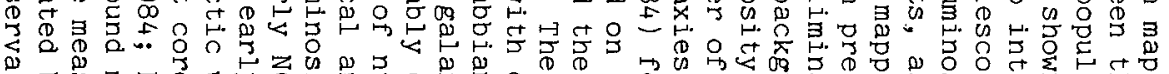

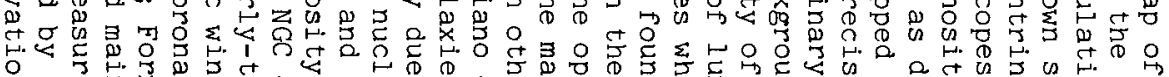

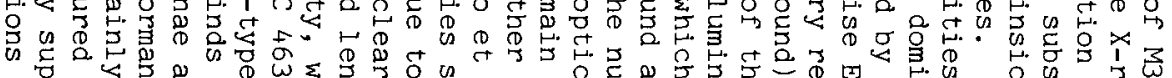

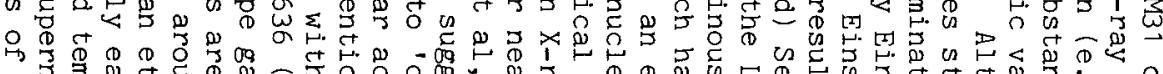

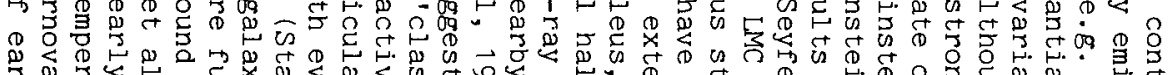
马山今心

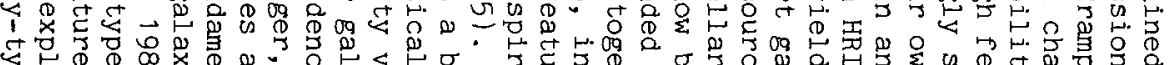

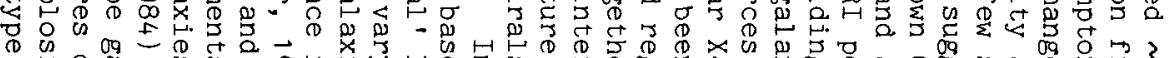

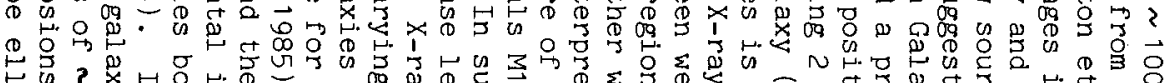

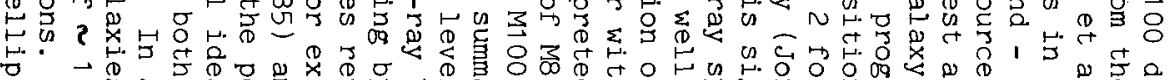
萿

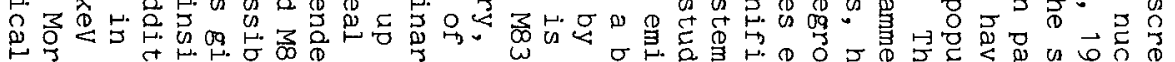
क क U

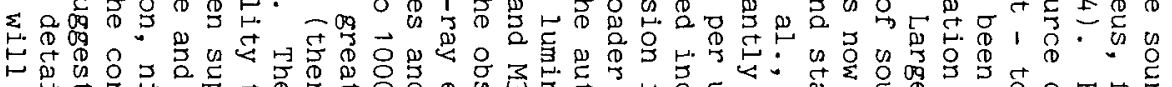

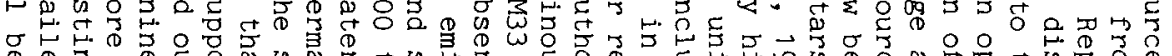

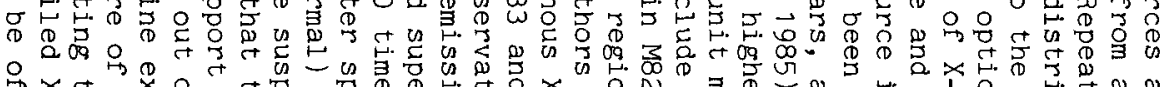

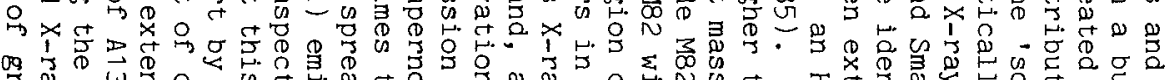

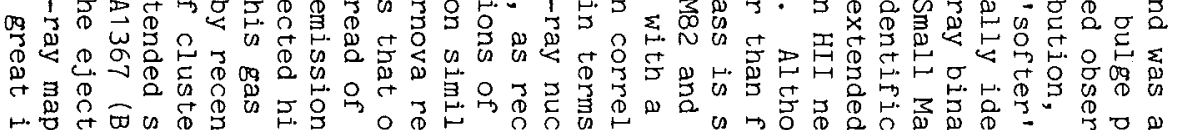

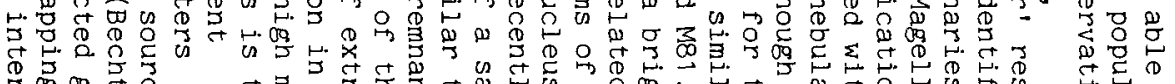

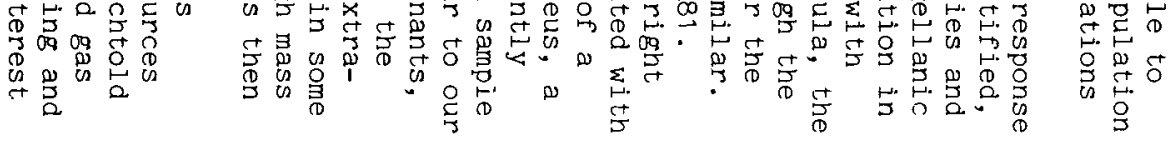

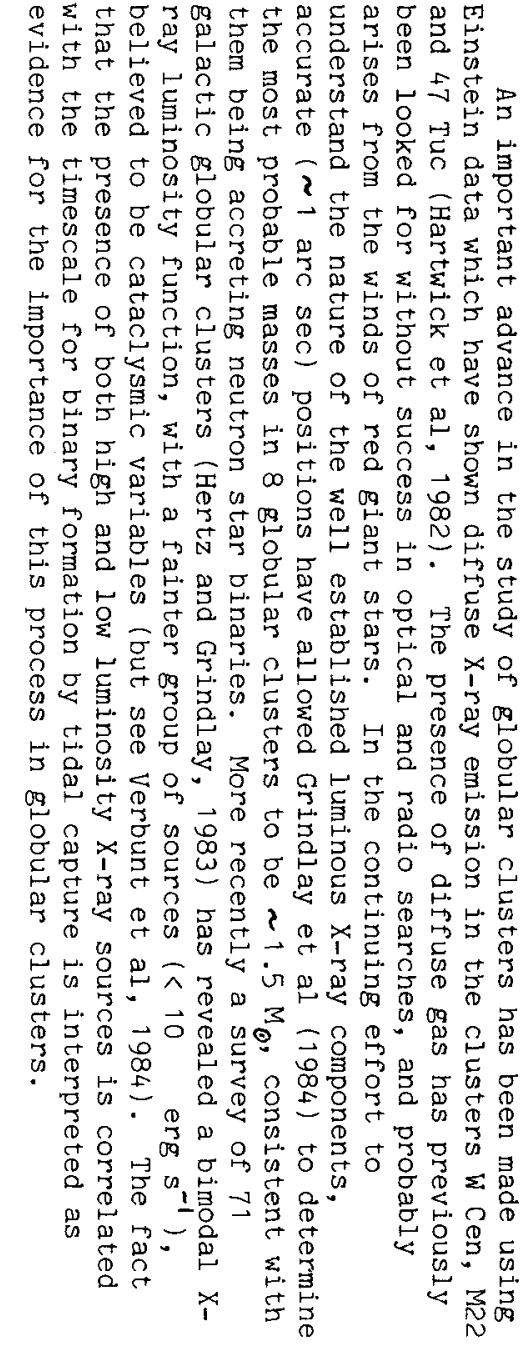


in determining the amount of hot gas, the heating mechanism, extension, and confining medium (dark halo?) and whether or not (as seems likely in NGC 4636) cooling flows are important in the central regions of the galaxy. On this topic, also see Nulsen et al. (1984).

(vi) Active galactic nuclei (AGN)

Substantial progress in analysing well-exposed Einstein fields (the Medium Deep Survey) has been made and Gioia et al (1984) recently reported the results from 150 fields covering 40 square degrees of sky. Approximately half of the 112 new X-ray sources are identified with AGN. The rapidly increasing number of AGN identifications will be of great value in improving both the AGN luminosity function(s) and estimates of their contributions to the X-ray background.

Broad-band X-ray spectra have been reported for a sample of the brighter AGN, covering a wide range of luminosity ( $\left.\sim 10^{42}-10^{46} \mathrm{erg} \mathrm{s} \mathrm{s}^{-1}\right)$ and morphological type and Mushotzky (1984), in reviewing these mainly HEAO-1 and Einstein MPC and SSS data, reports a remarkable uniformity of spectral slope. New EXOSAT spectra, reviewed by Pounds et al. (1985), confirm many of these 'canonical' spectra (photon number index $\sim 1.7$ ) but also provide evidence for several steeper spectra and for spectral variability. Two particularly interesting results from this EXOSAT study are the discovery of a soft spectrum and marked variability (on a timescale $\sim 1$ hour) for the nearby seyfert galaxy NGC 4051. The EXOSAT observations of steep, soft $X$-ray components in several $A G N$ conforms with a result from the re-analysis of Einstein IPC spectra of the brighter QSOS (Elvis, private communication) and may correspond to the spectral break 'required' to join the X-ray and UVOIR continua of AGN. Einstein (Holt et al, 1980) and EXOSAT (Pounds, 1985) observations of NGC 4151 have shown the potential of X-ray spectra for studying the environment of AGN, both analyses providing evidence for a complex absorbing structure in the surrounding gas. A particularly interesting spectral feature was detected in the Einstein OGS observation of the BL Lac object PKS 2155-304. An absorption edge or trough at $\sim 600 \mathrm{eV}$, probably attributable to ionised oxygen in the vicinity of the AGN or in an (hypothesised) intervening intergalactic medium (Canizares and Kruper, 19841 .

\section{(vii) Clusters of galaxies}

Analysis of the rich Einstein data base has continued, extending the range of cluster morphologies and demonstrating a continuous distribution of loose groupings to highly evolved clusters (Jones and Forman, 1984). The Einstein observations also show that many of the clusters with a dominant central galaxy probably have central cooling flows, which could contribute substantially to the growth of this central galaxy. From a determination of the central density and temperature of 36 clusters of galaxies, stewart et al. (1984) estimated cooling times less than the Hubble time and derived mass inflow rates of between 2 and $400 \mathrm{M}_{\mathscr{O}} \mathrm{yr}^{-1}$. The search for distant clusters $(\mathrm{Z}>0.5)$ is important in relation to the fundamental questions of cluster formation and evolution. X-ray observations should prove an effective way of discovering very distant clusters, due to their characteristic extended emission and lower confusion level (than in the optical or radio bands). McHardy and Cooke at Leicester have been using Einstein and EXOSAT to search for X-ray emission from suspected distant clusters, selected from a sample of $\sim 150$ steep spectrum radio sources with deep CCD optical follow-up, while Harris (CFA) is studying serendipitous Einstein sources for the same purpose.

(viii) Gamma ray bursts

Identification and understanding of the intense (but rare) $\gamma$-ray bursts has 
been brought closer by the determination of several relatively precise source positions. Cline et al (1984) have reported positions accurate to a few (arc min) for the moderately intense bursts of 1978 Nov 24 and 1979 Nov 5 and 16 , from the interplanetary network of Soviet, US and European spacecraft. Although all 3 locations are at high galactic latitude none have an obvious steady optical counterpart; however, a search of archived data produced a coincident optical event, confirming the previous $\not$-ray burst/optical transient association discovered by Schaefer (Schaefer, 1981). Barat et al (1984) report the precise location of the intense 1979 January $13 \gamma$-ray burst, probably coincident with a second of the Schaefer et al (1984) optical flashes and with a possible faint $\left(m_{v} \sim 23\right)$ quiescent optical counterpart. A quasi-periodic repetition of $x-$ ray pulses makes this observation different to most previous detections. On the basis of a total of 3 optical flashes seen near $\gamma$-ray burst locations, Schaefer et al (1984) conclude an average recurrence timescale of $\sim 1$ year and a ratio of $\gamma$-ray to optical flux of $\sim 900$. Although the $x$-rays burst sources remain an unsolved puzzle, these new data appear to support the view that they originate within the magnetosphere or at the surface of an isolated or weakly accreting neutron star.

\section{FUTURE PROSPECTS}

By its nature, the Einstein Observatory examined only a small fraction of the whole sky, an aspect in common with most large ground-based telescopes. The German ROSAT mission, due for launch on the US Space Shuttle in late 1987, will carry a somewhat larger Wolter I telescope, of similar properties to Einstein (see Table), which will be used in the first $\sim 6$ months in orbit to conduct an all-sky survey with a sensitivity at least comparable to the Einstein mediumdeep survey (Trumper 1984). More than $10^{5}$ sources of all astronomical types are anticipated from this survey, greatly increasing the statistical power of many varied studies and - potentially - uncovering major new phenomena. Alongside the ROSAT X-ray telescope will be flown the UK Wide Field Camera, a telescope optimised for the extreme ultraviolet waveband (Pye, 1984). Following a simultaneous all-sky survey (probably the first in the XUV band), the WEC and ROSAT X-ray telescopes will then embark on a programme of observations of selected fields, in the manner of the Einstein Observatory. US astronomers will participate strongly in this phase of the mission.

Several months before the launch of ROSAT, the successor to Tenma is due to be placed in orbit by the Institute of Space and Astronautical Science. This Japanese satellite, ASTRO-C, will carry a 0.5 square metre array of proportional counter detectors, to conduct detailed timing and spectral studies of a wide range of galactic and extragalactic sources. UK astronomers are collaborating with ISAS on the payload development and will share in the scientific operation of ASTRO-C. This mission, complementary in its capabilities to ROSAT, will provide a powerful extension to the studies now being conducted with Tenma and EXOSAT (due to re-enter the atmosphere in mid-1987).

Beyond 1987, NASA plan to fly two satellites in the Explorer programme which cover this field of astronomy. The Extreme Ultra Violet Explorer (EUVE) will carry out a sky survey in the XUV band, similar to the ROSAT WFC, and may also have some additional spectroscopic capability. Later on, the X-ray Timing Explorer (XTE) is due for launch, carrying a powerful array of detectors, including a 1 square metre proportional counter array, for timing and spectral studies of the brighter galactic and extragalactic sources. In Italy, approval has recently been obtained for the SAX mission, an X-ray satellite to be launched on the US Space Shuttle towards the end of the $1980 \mathrm{~s}$ and designed to carry out a range of spectroscopic and hard $X$-ray mapping studies. Collaboration of Dutch astronomers is proposed for SAX. Finally, in the present decade, it is hoped that a small Indian X-ray payload will be launched, while 
further experiments are planned in the Russian programme (though details are not yet known). In summary, it appears that the 1980 s should see a total, worldwide activity in X-ray astronomy similar to that of the previous decade, but with a much broader international spread.

TABLE

Characteristics of some recent and planned X-ray telescopes

\begin{tabular}{|c|c|c|c|c|c|}
\hline Telescope & Einstein & EXOSAT & ROSAT XRT & ROSAT WEC & AXAF \\
\hline Type & Wolter I & Wolter I & Wolter I & $\begin{array}{l}\text { Wolter- } \\
\text { Schwarzschild I }\end{array}$ & Wolter I \\
\hline Nested shells & 4 & 2 & 4 & 3 & 6 \\
\hline Focal length $(\mathrm{cm})$ & 344 & 109 & 240 & 52 & 1000 \\
\hline Max. diameter $(\mathrm{cm})$ & 58 & 28 & 83 & 58 & 120 \\
\hline Geom. area $(\mathrm{cm})$ & 350 & 89 & 1150 & 511 & 1500 \\
\hline Energy range (keV) & $0.2-4$ & $0.04-1.5$ & $0.1-2$ & $0.015-0.2$ & $0.1-10$ \\
\hline \multicolumn{6}{|c|}{ On-axis resolution (arc $\mathrm{sec}$ ) } \\
\hline EWHM & 3 & 5 & 5 & 30 & 0.5 \\
\hline HER $(44$ A) & 4 & 10 & 2.5 & 60 & 0.5 \\
\hline HER ( 8 A) & 5 & 15 & 2.5 & 60 & 0.5 \\
\hline
\end{tabular}

In the 1990s, further Japanese and European X-ray missions (ASTRO-D and XMM, respectively) are firmly planned, very probably with an emphasis on realising the great scientific potential of moderate-to-high resolution $\mathrm{X}$-ray spectroscopy. A major focus will be on the NASA AXAF mission. This natural (US) successor to the Einstein Observatory, with a nested Wolter I telescope of maximum mirror diameter 1.2 metres and sub-arc second resolution, is seen as the $X$-ray counterpart to the Hubble Space Telescope and will be the most powerful Xray mission yet conceived.

\section{REFERENCES}

Andrews, M., Basart, J., Lamb, R. and Becker, R. (1983) Ap. J. 266, 684. Arnaud, M., Ballet, J., Rocchia, R. and Rothenflug, R. (1985) Proc. 18th ESLAB Symposium on X-ray Astronomy (Reidel).

Aschenbach, B. et al., (1985) To be published.

Barat, C. et al. (1984) Ap. J. 286, L5.

Barstow, M.A., (1985) Proc. 18th ESLAB Symposium on X-ray Astronomy (Reidel).

Bechtold, J. et al. (1983) Ap. J. 265, 26.

Becker, R., Helfand, D. and Szymkowiak, A. (1982) Ap. J. 255, 557.

Bradt, H. and McClintock, J.E. (1983) Ann. Rev. Astron. and Ap. 21, 13.

Caillault, J.P. and Helfand, D. (1982) B.A.A.S. 13, 811.

Caillault, J.P. and Helfand, D.J. (1985) Ap. J. 289 , in press.

Canizares, C.R. and Kruper, J. (184) Ap. J. 278, L99.

Charles, P.A. et al. (1983) Mon. Not. R. astr. Soc. 202, 657.

Cline, T.L. et al. (1984) Ap. J. 286, L15.

Cominsky, L.R. and Wood, K.S. (1984) Ap. J. 283, 765.

Cordova, F.A., Chester, T.J., Mason, K.O., Kahn, S.M. and Garmire, G.P. (1984) Ap. J. 278,739 .

Cordova, F.A. and Mason, K.O. (1983) Ch. 4 in "Accretion driven stellar X-ray 
sources" (C.U.P.) Eds. Lewin and van den Heuvel.

Courvoisier, T., Parmar, A. and Peacock, A. (1985) Proc. 18th ESLAB Symposium on $\mathrm{X}$-ray Astronomy (Reidel).

Cowley, A.P., Crampton, D. and Hutchings J.B. (1982) Ap. J. 255, 596.

Crampton, D., Cowley, A.P., Hutchings, J.B., Schade, D.J. and van Speybroeck, L.P. (1984) Ap. J. 284, 663.

Culnane, J.L., White, N.E., Kahn, S., Parmar, A.N., Blissett, R.J. and Kellett, B. (1985) Proc. 18th ESLAB Symposium on X-ray Astronomy (Reidel).

Fabbiano, G., Fransson, C. Palumbo, G.C. and Trinchieiri, G. (1985) Proc. 18th ESLAB Symposium on X-ray Astronomy (Reidel).

Fabian, A.C., Hu, E.M. Cowie, L.L. and Grindlay, J. (1981) Ap. J. 248, 47.

Fahlman, G.G. and Gregory, P.C. (1983) in "Supernova remnants and their X-ray emission" (Reidel) Eds. Danziger and Gorenstein, P445.

Forman, W., Jones, C., \& Tucker, W. (1984) in "Large Scale Structure of the Universe" Proc. Trieste Meeting.

Gioia, I.M., et al. 1984) Ap. J. 283, 495.

Grindlay, J.E. et al. (1978) Nature 274, 567.

Grindlay, J.E., Hertz, P., Steiner, J.E., Murray, S.S. and Lightman, A.P. (1984) Ap. J. 282, L13.

Hartwick, F., Cowley, A. and Grindlay, J. (1982) Ap. J. 254, L11.

Hertz, P. and Grindlay, J.E. (1983) Ap. J. 275, 105.

Holt, S.S. (1983) "Supernova remnants and their X-ray emission" (Reidel) Ed. Danziger and Gorenstein, P17.

Holt, S.S. et al. 1980) Ap. J. 241 , L13.

Horstman, H., Balunias, S., Cecchini, S., Palumbo, C.G. Ramond, J.C. and Rosso C. (1985) Proc. 18th ESLAB Symposium on X-ray Astronomy (Reidel).

Jones, C. and Forman W. (1984) Ap. J. 276, 38.

Jones, L.R., Pye, J.P. and Culhane, J.L. (1984) Proc. Bologna Symposium on Xray Astronomy - to be published by ISAS.

Jones, L.R., Pye, J.P. and McHardy, I.M. (1985) Proc. 18th ESLAB Symposium on Xray Astronomy (Reidel).

Kahn, S.M., Wesemael, F., Liebert, J., Raymond, J.C., Steiner, J.E. and Shipman, H.L. (1984) Ap. J. $278,255$.

Kellett, B.J. (1985) Proc. 18th Symposium on X-ray Astronomy (Reidel).

Kelley, R.L., Jernigan, J.G., Levine, A., Petro, L.D. and Rappaport, S. (1983) Ap. J. 264,568 .

King, A.R. (1985) Proc. 12th Texas Symposium on Relativistic Astrophysics (N.Y. Acad. Sci.)

Ku, W.H.M., Kann, S.M., Pisarski, R.L. and Long, K.S. (1984) Ap. J. 278, 615.

Lewin, W.H.G. and van den Heuvel, E.P. (1983) Eds. of "Accretion driven stellar X-ray Sources" (C.U.P.).

Lucy, L.B. and White, R. L. (1980) Ap. J. 241, 300.

Lucy, L.B. (1982) Ap. J. 255, 286.

McClintock, J.E. et al. (1982) Ap. J. 258, 245.

Maejima, Y., Makishima, K., Matsuoka, M., Ogawara, Y. and Oda, M. (1984) Ap. J. 285,712 .

Mandelstam, S.L. et al. (193) Adv. Space. Res. 2, 293.

Mathewson, D. et al. (1983) "Supernova remnants and their X-ray emission"

(Reidel) Eds. Danziger Gorenstein, P541.

Matsuoka, M. et al. (1984) Ap. J. 283, 774 .

Meekins, J.F. et al. (1984) Ap. J. 278, 288.

Mewe, R. et al. (1981) in 'Cambridge Cool Star Workshop' Ed. Dupree.

Montmerle, T., Koch-Miramond, L., Falgarone, E. and Grindlay, J.E. (1983) Ap. J. 269,182 .

Motch, C. Pedersen, H. Ilovaisky, S.A., Chevalier, C. and Pakull, M.W. (1984)

Proc. Bologna Symposium - to be published by ISAS.

Mushotzky, R.F. (1984) NASA Tech. Memo. 86071.

Nagase, F. et al. (1984) Ap. J. 280, 259.

Nulsen, P.E.J. et al. (1982) Mon. Not. R. astr. Soc. 199, 1089. 
Nulsen, P.E.J., Stewart, G.C. and Fabian, A.C. (1984) Mon. Not. R. astr. Soc. 208, 185.

Page, C.G. (1985) Proc. 18th ESLAB Symposium on X-ray Astronomy (Reidel).

Parmar, A.N., Culhane, J.L., White, N.E. and van den Oord, G.H.J. (1985) Proc. 18 th ESLAB Symposium on X-ray Astronomy (Reidel).

Pedersen, H., van Paradijs, J, and Lewin, W.H.G. (1981) Nature 294, 725.

Petre, R., Canizares, C.R., Kriss, G.A. and Winkler, P.F. (1982) Ap. J. $258,22$.

Pisarski, R.L., Helfand, D.J. and Kahn, S.M. (1984) Ap. J. 277, 710.

Pounds, K.A. (1984) Proc. Bologna Symposium on X-ray Astronomy - to be published by ISAS.

Pounds, K.A. Warwick, R.S., Culhane, J.L. and de Korte, P. (1985) Proc. 18th ESLAB Symposium on X-ray Astronomy (Reidel).

Pye, J.P. and McHardy I.M. (1983) Mon. Not. R. astr. Soc. 205, 875.

Pye, J.P. (1984) MPE Report 184 "X-ray and UV emission from AGN". Eds Brinkman and Trumper P261.

Pye, J.P. Pounds, K.A., Rolf, D.P., Seward, F.D., Smith, A. and Willingale, R. (1981), Mon. Not. R. astr. Soc. 194, 569.

Rappaport, S. and ven den Heuvel, E.P. (1982) Proc. IAU Symposium 98 on "Be Stars" (Reidel) Eds. Jaschek and Groth. p327.

Remillard, R.A. and Canizares, C.R. (1984) Ap. J. 278, 761.

Schaefer, B.E. (1981) Nature 294, 722 .

Schaefer, B.E. et al. (1984) Ap. J. 286, L1.

Schmitt, J. Golub, L., Harnden, F.R., Maxson, C.W., Rosner, R. and Vaiana, G. (1985) Ap. J. to be published.

Seward, F.D. (1983) in "Supernova remnants and their X-ray emission" (Reidel) Eds. Danziger and Gorenstein, P405.

Seward, F.D., Gorenstein, P. and Tucker, W. (1983) Ap. J. 266, 287.

Seward, F.D. and Harnden, F.R. (1982) Ap. J. 256 , L45.

Stanger, V. J. (1985) Ph. D. Thesis, University of Leicester.

Steiner, J.E. et al. (1984) Ap. J. 280, 688.

Stern, R.A., Zolcinski, M.C., Antioches, S.K. and Underwood, J.H. 1981) Ap. J. $249,647$.

Stewart, G.C., Fabian, A.C. and Seward, F.D. (1983) "Supernova remnants and their X-ray emission" (Reidel) Ed. Danziger and Gorenstein.

Swank, J. and Carsinelli J.P. (1983) Ap. J. $271,681$.

Swank, J. and Johnson, H.M. (1982) Ap. J. 259, L67.

Swank, J., White, N.E., Holt, S. and Becker, R. 1981) Ap. J. 246, 208.

Tuohy, I. and Garmire, G. (1980) Ap. J. 239, L107.

Trumper, J. (1984) MPE Report 184 "X-ray and UV emission from AGN". Eds. Brinkman and Trumper.

Turner, M.J.L. and Breedon, L.M. (1984) Mon. Not. R. astr. Soc. 208, 29 P.

Vaiana, G.S. et al. et al, (1981) Ap. J. 245, 163.

Vedder, P.W. and Canizares, C.R. (1983) AP. J. 270, 666.

Verbunt, F., van Paradijs, J. \& Elson, R. (1984) Mon. Not. R. astr. Soc. $210,899$. Vilhu, O. Heise, J. (1985) Proc. 18th ESLAB Symposium on X-ray Astronomy (Reidel). Waki, I., Inoue, H., Koyama, K. et al. (1984) Pub. Astron. Soc. Japan - in press. Waldron, W.L. (1981) U. Wisconsin, Thesis.

Warwick, R.S. et al. (1981) Mon. Not. R. astr. 197, 865.

Watson, M.G., King, A.R. and Heise, J. (1985) Proc. 18th ESLAB Symposium on Xray Astronomy (Reidel).

Watson, M.G., King, A.R. and Osborne, J. (1985a) Mon. Not. R. astr. Soc.

Watson, M.G., Stanger, V.J. and Griffiths, R.E. (1984) Ap. J. 286, 144.

Watson, M.G. Warwick, R.S. and Ricketts, M.J. (1981) Mon. Not. R. astr. Soc. 195, 197.

White, N.E. Parmar, A.N., Sztajno, M. and Zimmermann, H., Mason, K.O. and Kahn, S.M. (1984) Ap. J. 283, L9.

White, N.E. and Swank, J. (1982) Ap. J. 253, L61.

Winkler, P.F., Canizares, C.R., Clark, G.W., Markert, T.H., Kalata, K. and Schnopper, H. (1981) Ap. J. 246, L27. 